\title{
CARRÉ ROUGE SUR FOND NOIR. \\ LE POLITIQUE COMME VOLONTÉ \\ ET REPRÉSENTATION
}

\author{
GAGNON, ALEX. \\ Candidat au doctorat, Département des littératures de langue française, Université de Montréal
}

Résumé: Le présent article vise à opérer, délibérément et à des fins ouvertement politiques, une (re)construction des événements du printemps 2012 sur la base d'un ensemble de concepts empruntés, notamment, à une certaine philosophie politique. II s'agit d'abord de mettre en évidence le fait que toute lutte sociopolitique est d'abord une lutte située dans le champ du langage et des significations. Sur cette base, l'auteur propose d'envisager les événements du printemps 2012 comme une « crise politique » : le choix de cette appellation ne traduit pas un constat, mais bien une stratégie d'analyse (le choix d'une perspective parmi d'autres possibles) visant à attirer l'attention sur ce qui a peut-être, en somme, constitué l'enjeu le plus profond du conflit, à savoir la définition même de la « démocratie » - les camps adverses revendiquant l'un comme l'autre leur appartenance à cet incontournable mot, dont ils prétendent avoir la définition légitime.

Mots-clés : Grève étudiante, démocratie, désobéissance civile, théories politiques, rhétorique

\begin{abstract}
Drawing on a group of concepts from a certain political philosophy, this paper seeks, deliberately and with overt political intentions, a (re)construction of the events surrounding the spring of 2012. This first means putting emphasis on the fact that any socio-political struggle is primarily situated in the field of language and meaning. Accordingly, the author suggests thinking the events of the spring of 2012 as "political crisis": a choice of words that does not translate as a statement, but rather as an analytical strategy (the choice of one perspective among many) aimed at bringing the attention on what has maybe, in short, constituted the core of the conflict, that is, the act of defining "democracy" - with both opposing parties claiming their affiliation to this unavoidable word, to which they pretend having the most legitimate definition.
\end{abstract}

Keywords: Student strike, democracy, civil disobedience, political theory, rhetoric 
Ces étudiants actifs politiquement, qui viennent assez souvent des sciences sociales ou des disciplines philologiques et historiques, présentent une certaine immunité vis-à-vis de la conscience technocratique, car dans les deux cas et même si c'est pour des motifs différents, l'expérience qu'ils ont de leur propre travail scientifique est en contradiction avec les hypothèses qui sont au fondement de la conscience technocratique.

Jürgen Habermas, La technique et la science comme idéologie

La mobilisation étudiante du printemps 2012, à travers les discours qu'elle a produits, s'est ellemême déclarée " historique ». Pareille historicisation du présent, qui serait tributaire, selon François Hartog, d'une économie médiatique où la conscience du futur se traduit par l'usage au présent du futur antérieur, donc dans laquelle l'événement tend à se retourner «sur lui-même pour anticiper le regard qu'on portera sur lui » (Hartog, [2003] 2012 : 158), a ceci de particulier qu'elle est liée à une tentative des actrices et acteurs sociaux de construire discursivement la grandeur d'un événement en fabriquant, précisément, son statut d'« événement ». À la manière d'un performatif, cette historicisation tente de faire advenir ce qu'elle énonce par son énonciation elle-même. Elle tente de produire l'événement avant même que la médiation en quoi consistent le discours et la distance historiographiques ait pu, pour parler comme Paul Veyne, parcourir et appréhender l'ensemble du " champ événementiel ${ }^{1}$ » qui donnera éventuellement à la grève étudiante sa figure et son sens historiques (Veyne, $1971: 51)^{2}$. De ce point de vue, la survenue de l'événement, qui cherche à dire et à justifier son propre avènement, est toujours déjà sa représentation. Et en effet, dans la volumineuse masse de discours suscitée par la mobilisation qu'a portée et supportée la grève étudiante, la part occupée par le métadiscursif, donc par la dimension autoréflexive, est assurément significative. À telle enseigne qu'elle semble avoir été constitutive du mouvement lui-même, inhérente à son expansion.

Pourtant, de cette foisonnante végétation discursive qu'a produite la mobilisation étudiante, de cet espace de créativité langagière, de cette importante prolifération de paroles sur la grève et ses enjeux n'émerge encore aucune analyse politique approfondie, aucun bilan réflexif provisoire. Provisoire parce qu'en effet, en un certain sens, rien n'est encore terminé. Provisoire parce qu'il faut absolument se garder de muséifier une fois pour toutes le déroulement et le sens des événements.

1. Dans le cadre de l'épistémologie de l'histoire développée par Paul Veyne, le « champ événementiel » désigne la nappe objective et multiple des faits et des discours qui précède l'intervention historienne de la mise en intrigue, donc du récit historiographique. L'idée de " champ événementiel » implique donc que " l'événement n'est pas un être, mais un croisement d'itinéraires possibles » (Veyne, 1971: 51).

2. C'est en vertu de l'aspect narratif du travail historiographique que la signification d'un événement, partiellement extrinsèque à l'événement lui-même, ne peut advenir qu'après cet événement ou, comme le mentionne Paul Ricoeur, « qu'à la lumière d'événements futurs » (Ricoeur, 1983 : 261). La narration historiographique constitue un récit que les narratologues appelleraient ultérieur : en ce sens, «il n’y a pas d'histoire du présent », et si le présent s'insère dans une histoire, il ne forme pourtant pas encore un récit (Ricoeur, 1983 : 262). Mais cette affirmation, pour demeurer valable et nécessaire sur le plan théorique, n'invalide aucunement la valeur polémique circonstancielle de cette " auto-historicisation » discursive du mouvement étudiant : il s'agissait, dans le contexte d'énonciation, de légitimer la mobilisation pour répondre à un véritable déni de reconnaissance. 
Certes, on ne saurait le reprocher à qui que ce soit ; mais il est un terrain, encore inoccupé, que le discours devra impérativement investir. Car on ne trouve encore aucune tentative de synthèse qui, informée par des considérations à la fois sociologiques et philosophiques, s'efforcerait de jeter les bases d'une réflexion critique générale et englobante sur la nature proprement politique des événements du printemps 2012. Le présent article trouve son origine et son lieu dans cette brèche étonnante. Car la performativité évoquée plus haut, par laquelle le mouvement étudiant s'est autoconsacré comme fait historique, est nécessairement fragile. En prétendant forcer les portes de I'histoire, le mouvement ne fait encore rien d'autre qu'une demande : il demande à être pensé, appelle un prolongement et des effets tout en exigeant une réflexion sur le politique qui serait apte à en recueillir, pour alimenter une mémoire collective, les enseignements. Autrement dit, le fait que le mouvement se soit autoproclamé historique ne doit pas faire oublier qu'il ne l'est pas nécessairement encore et qu'il demande simplement à le devenir, bref que son statut et sa signification d'événement historique ne sont pas encore définitivement cristallisés. Et c'est précisément parce que son sens est encore loin d'être sédimenté que cet article prétend pouvoir participer au processus de sa sédimentation. Lecteurs et lectrices ne trouveront pas ici la forme finale et définitive de cette réflexion politique appelée par la crise étudiante; ils et elles trouveront plutôt, sous la forme d'un premier déblayage, l'amorce de cette réflexion, c'est-à-dire l'esquisse des contours qu'elle pourrait prendre et des lignes directrices qu'elle pourrait suivre.

L'idée que, dans les pages qui suivent, j'aimerais défendre et explorer peut se ramener à un énoncé relativement simple : la mobilisation étudiante me paraît avoir joué le rôle d'un prisme à travers lequel la chose politique elle-même s'est trouvée à la fois mise en abyme et mise en relief. Dans les circonstances actuelles, il importe de partir de ce constat initial si nous souhaitons éviter de manquer à celle parmi nos tâches qui consiste non seulement à produire du sens à partir des signes qui nous entourent, mais encore à prévenir l'écueil que serait une « décollectivisation » des suites du printemps 2012 - sous la forme, par exemple, d'une juxtaposition de mémoires individuelles plus ou moins empreintes d'un certain romantisme. II s'agit donc, en un mot, de penser la crise pour la soustraire à deux forces, à deux emprises qui tendent à la rendre inoffensive. II faut l'arracher, d'une part, à une culture du spectacle qui opère généralement dans le sens de ce que Pierre Bourdieu appelle, lorsqu'il analyse les effets sociaux des images médiatiques, la " dramatisation ", au double sens d'une mise en scène et d'une concentration du sensationnel (Bourdieu, 2008 : 18). Il faut également la soustraire, d'autre part, au risque incessant de sa récupération par un langage politique qui cherche à réduire les enjeux du débat au seul problème de la gestion et du financement des universités. Autour de ce problème et de part et d'autre de la clôture idéologique, le discours des chiffres, le traitement des données, la multiplication des analyses localisées et l'écran que forment les innombrables référents économiques ont (eu) pour effet d'alimenter cette « contradiction originale» qui constitue, pour Marcel Gauchet, l'une des conditions de la démocratie contemporaine : " une société qui se sait incomparablement dans son détail sans se comprendre dans son ensemble » (Gauchet, 1998 : 174). 


\section{SITUATION DU PROBLÈME. LE POLITIQUE ET LA POLITIQUE}

Qu'est-ce que les événements du printemps 2012 nous permettent de comprendre et de dire sur la chose politique? Quels sont les problèmes que ces événements nous autorisent à formuler sur les jeux et les enjeux de pouvoir dans une société qui cherche tant et tellement à se comprendre elle-même par l'entremise du concept de « démocratie »? Telles sont les questions générales qui méritent ici d'être posées.

Le concept de "démocratie », tel qu'il fonctionne aujourd'hui, apparaît comme le produit d'une histoire complexe. Relativement stable jusqu'à la fin du xviiie siècle, où il conserve encore généralement le sens que lui donnaient les Anciens - tandis que l'assemblée du peuple réunie, c'est-à-dire plus précisément de la frange politiquement reconnue de celui-ci, se voit reconnaître un rôle institutionnel direct -, le concept subira, sous le coup des révolutions américaine et française et des situations politiques qui en découlent, une transformation sémantique radicale. II fera l'objet, au xix siècle, d'une étonnante appropriation par les tenants d'un système électoral théoriquement fondé sur le principe de représentation. Depuis cette époque, la signification du régime « démocratique », flottante et conflictuelle, est perméable aux multiples interprétations, embrouillée par la généralisation du mot lui-même. Et à telle enseigne que, dans les sociétés modernes, le consensus sur son usage et le dissensus sur sa définition semblent parfaitement corrélatifs. Ainsi François Guizot pouvait-il écrire, en 1849, du mot « démocratie » qu'il était devenu « le mot souverain, universel. Tous les partis l'invoquent et veulent se l'approprier comme un talisman » (cité dans Dupuis-Déri, 2013 : 349). Et comme le remarque à juste titre la philosophe politique Chantal Mouffe, cette extension sémantique et pragmatique de la notion caractérise également nos sociétés contemporaines : "Le signifiant démocratie, écrit-elle, fonctionne maintenant comme horizon imaginaire où viennent s'inscrire des revendications extrêmement disparates, et le consensus qu'il semble indiquer risque bien d'être une illusion » (Mouffe, 1995 : 120).

Cette constatation est fondamentale puisque qu'elle enracine les luttes politiques dans le champ des significations, et plus précisément parce qu'elle loge la chose politique dans cet espace de tensions qui se creuse entre la stabilité des signifiants et l'instabilité des signifiés. II n'y a de luttes politiques que dans et par le langage, et en ce sens la condition politique, depuis que le fondement et la justification du pouvoir et de la société échappent au principe divin, réside dans une indécidabilité de la signification. C'est ce que voyait bien Fernand Dumont lorsqu'il faisait correspondre, dans Le Lieu de l'homme, le régime démocratique avec une « diversification des lieux d'où le sens qui advient à l'histoire prête à lecture » (Dumont, [1968] 2005 : 265). Dès lors, il devient réducteur, d'un point de vue rétrospectif, de faire des droits de scolarité l'unique point de litige de la grève étudiante et de reléguer les autres problèmes soulevés par la mobilisation dans le domaine du périphérique ou de l'épiphénomène, bref d'opérer une démarcation de principe entre l'enjeu instigateur d'une lutte politique et les moyens de lutter, à la fois pratiques et sémantiques, mis en œuvre dans le cadre de cette lutte. Car à force d'étalement et de radicalisation, la mobilisation étudiante est devenue le théâtre non seulement d'un conflit sur le sens à donner aux institutions universitaires québécoises, mais encore d'une lutte pour la définition légitime du concept de " démocratie », convoqué de part et d'autre, revendiqué à gauche comme à droite, célébré en haut comme en bas. 
Or, cela fait signe vers deux réalités sociologiques de tout mouvement de contestation. D'une part, comme l'indique l'analyse bourdieusienne, il est légitime de supposer que tout conflit social et politique qui perdure donne lieu, de façon presque inéluctable, à une démultiplication (ou à un redoublement) des enjeux de la lutte à travers laquelle les moyens de lutter ainsi que la définition des enjeux et de la lutte elle-même deviennent de nouveaux enjeux de lutte. Bourdieu souligne que, "dans ce contexte, la distinction entre des grèves politiques et des grèves non politiques », ou par exemple, entre une " grève » et un «boycott», est une stratégie intéressée, c'est-à-dire une «manipulation politique de la définition du politique » (Bourdieu, [1984] 2002 : 258). Autrement dit, l'expansion d'un mouvement de contestation risque toujours de se traduire par une extension de ses enjeux. D'autre part, toute contestation politique importante révèle une crise du langage et est elle-même, en son fondement, une lutte qui s'installe dans le champ des significations. C'est en ce sens, notamment, que le même Bourdieu parle de la « lutte des classements », un biais par lequel, poursuit-il, « la production symbolique intervient dans la lutte politique» (Bourdieu, [1984] 2002 : 62). On rejoint ici la liaison intime entre politique et rhétorique telle que la philosophie occidentale n'a jamais cessé de la développer, et telle qu'il faut la reprendre, comme cadre heuristique, pour explorer l'idée d'une coextensivité entre champ politique et champ du langage. Car si toute lutte politique est, constitutivement, une lutte pour le pouvoir de définition des mots et des notions avec lesquels on cherche à donner sens au monde social, alors le langage est, par excellence, ce lieu où vient s'inscrire le politique.

«Penser » la crise du printemps 2012, et penser la crise comme un tout, implique que l'on se réapproprie, j'oserais dire «épistémologiquement », les événements concernés. Et si je convoque ici la notion de "crise ", c'est parce qu'elle me paraît être la seule capable de décrire et d'interpréter ces événements. En effet, l'expansion et l'extension du mouvement étudiant, dont il vient d'être question, peuvent être analysées comme les signes d'une « crise politique », idée qui demande à être construite à partir d'une double opération : une déconstruction de l'image dominante du conflit étudiant telle que produite par le discours médiatique ${ }^{3}$ et une reconstruction sur de nouvelles bases conceptuelles. Comme le rappelle Michel Freitag, le concept de crise « comporte nécessairement une double référence objective et subjective » (Freitag, [1995] 1998 : 85). La crise réfère à la fois, dans sa dimension objective, à un processus qui perturbe et menace un fonctionnement régulier, et dans sa dimension subjective à la réaction de celles et ceux qui en font l'épreuve en vue d'un redressement de situation. Plus précisément, elle renvoie au fait que certains rouages de la vie collective, et dont la vie collective suppose l'invisibilité au moins partielle, puissent se trouver, si j'ose dire, momentanément dénudés, exposés et questionnés. Une crise politique surgit donc là où, plus ou moins subitement, les actrices et acteurs sociaux se mettent à percevoir que la discussion politique sur certains enjeux se double d'une discussion portant sur les conditions généralement indiscutées de cette discussion politique.

3. À titre indicatif, le bilan que la firme Influence communication a rendu public en décembre 2012 indique que la couverture médiatique des événements a généralement repris la logique du « fait divers » au détriment des discussions portant sur les différents enjeux liés à la question des universités. Le document peut être consulté à l'adresse suivante : http://www.influencecommunication.com/content/etat-de-la-nouvelle-bilan-2012. On consultera aussi un article paru dans Le Devoir du 20 février 2013 au sujet d'une enquête menée par le Centre d'études sur les médias (Sauvageau et Thibault, 2013). 
Dans cette perspective, il semble que l'on puisse comprendre et analyser la crise étudiante comme ayant révélé ce qui, dans nos sociétés, distingue et relie d'un seul tenant le politique et la politique, si l'on accepte de donner à ce nœud conceptuel le sens que lui confère Chantal Mouffe. Alors que le politique " est lié à la dimension d'antagonisme et d'hostilité qui existe dans les rapports humains », la politique quant à elle "vise à établir un ordre, à organiser la coexistence humaine dans des conditions qui sont toujours conflictuelles car traversées par le politique» (Mouffe, 1994 : 101). Cette définition, qui reprend certains aspects de la conception arendtienne de la politique ${ }^{4}$, va plus loin que cette dernière et paraît, parce que plus précise, largement plus opératoire. Car dès lors que l'on distingue le politique de la politique, dès lors que l'on assigne à la seconde un rôle de régulation et au premier le statut d'élément constitutif des rapports sociaux, la tâche d'une politique démocratique peut et doit relever à la fois d'un travail de conversion et de conversation : il s'agit non pas d'« éliminer les passions ou de les reléguer à la sphère privée mais », plutôt, " de les mobiliser et de les mettre en scène selon des dispositifs agonistiques », donc de " transformer l'antagonisme en agonisme » (Mouffe, 1994 : 10-11). Cette transformation des « ennemi-e-s » en " adversaires » politiques doit s'effectuer par l'entremise des institutions, dont la fonction est non seulement de prendre en charge des valeurs " dans le cadre de leur reconnaissance collective ", mais aussi d'opérer une sorte de sublimation de la violence et, pour ce faire, d'aménager des espaces de discussion où les conditions mêmes de la discussion politique peuvent et doivent être discutées (Freitag, [1995] 1998 : 35). Autrement dit - si l'on reprend la définition de la "crise » envisagée plus haut -, les « dispositifs agonistiques » que Chantal Mouffe définit doublement comme conversion et conversation ont pour fonction d'institutionnaliser la crise. Devant une part irréductible d'antagonisme, qui forme au sein d'une société l'une des composantes intersubjectives de toute identification à des valeurs distinctives et à des communautés spécifiques, le rôle d'une politique démocratique serait de «multiplier les espaces où les rapports de pouvoir sont ouverts à la contestation » (Mouffe, $1994: 25$ )

\section{VOLONTÉ ET REPRÉSENTATION}

En rupture avec certaines théories politiques rationalistes, qui postulent l'existence d'une citoyenneté abstraite, et dont la prétendue universalité s'élabore traditionnellement sur la base d'une série d'exclusions ${ }^{5}$, le modèle théorique développé par Chantal Mouffe présente l'avantage indéniable de prendre en considération la structure particulière des sociétés contemporaines, où le pluralisme est une donnée capitale et où de nombreux mouvements sociaux s'articulent en fonction de la revendication d'identités sociales et culturelles locales et spécifiques. Par conséquent, ce modèle tient compte, contre une métaphysique du sujet pensé comme monade abstraite, du tissu de relations sociales plurielles hors desquelles il n'existe aucun sujet politique. II prend pour cadre d'analyse de la « démocratie » la dynamique de l'affrontement et de l'antagonisme, donc de la différence et du différend. Et dans la mesure où les événements du printemps 2012 nous font voir à quel point la vie en société nous place fondamentalement devant le fait d'avoir des ennemi-e-s, la théorie de la démocratie plurielle et radicale proposée par Chantal Mouffe fournit ici un ensemble 4. Arendt écrivait : « La politique prend naissance dans l'espace-qui-est-entre-les-hommes ... La politique prend naissance dans l'espace intermédiaire et elle se constitue comme relation » (Arendt, [1993] 1995 : 42).

5. Judith Butler écrivait aussi en ce sens que les exclu-e-s « constituent la limite contingente de l'universalisation » (Butler, 2004 : 129). C'est dire que « l'universel ne peut commencer à être formulé qu'à travers les défis lancés à sa formulation existante» (Butler, 2004 : 129). 
de balises pertinentes. Elle permet d'interpréter à la fois l'expansion prise par la mobilisation étudiante - qui n'a jamais cessé, en un certain sens, de demander aux représentant-e-s politiques qu'ils et elles jouent leur rôle en instituant des dispositifs agonistiques - ainsi que l'échec politique du gouvernement libéral de Jean Charest, qui s'accompagne d'une incapacité du discours public à constituer la grève étudiante comme véritable «crise politique ».

Cet échec se décline donc sur plusieurs plans d'analyse. Sur un premier plan, l'échec en est un au sens d'une conception agonistique de la démocratie. En effet, celle-ci exige que l'on reconnaisse, dès lors que la recherche et la formation de prétendus consensus impliquent nécessairement des formes de coercition ou d'exclusion plus ou moins dissimulées, l'existence irrémédiable d'un dissensus sur certaines valeurs à établir comme fondement des institutions et de la communauté. Alors que la logique de l'« agonisme » consiste à mettre en scène le dissensus et à le protéger de toute tentative de forclore la contestation, le comportement politique mis de l'avant par l'ancien gouvernement libéral a eu pour effet, sur la base d'une dénégation de la valeur du dissensus ${ }^{6}$, de révéler et de produire des polarisations tout en faisant durcir et proliférer les antagonismes. En ce sens, les événements du printemps 2012 offrent une prise de vue remarquable sur ce qui est susceptible de se produire lorsque la politique décide de s'exercer à partir d'une occultation du politique, lorsque la volonté politique s'autonomise radicalement par rapport aux contraintes de la représentation. Ce qui s'est joué, c'est en quelque sorte le refoulement du politique comme dimension propre et distincte des plans juridique et économique.

Dans une perspective historique, ce refoulement est révélateur d'une culture politique où, d'un côté, la fonction des gouvernements tend à être comprise et conçue comme relevant d'une forme de gestion pragmatique parmi tant d'autres et où, d'un autre côté, une conception généralement individualiste du droit tend à privilégier, comme mode de solution de crise, la « régulation juridique » au détriment de la «volonté politique » (Gauchet, 1998 : 119). Tout se passe comme si la vie politique, dans nos sociétés contemporaines, avait perdu le besoin de recourir, pour produire une justification du pouvoir et fonder la volonté d'agir, à une conception générale du monde et de l'être humain. La crise étudiante nous a placé-e-s devant un discours politique qui, d'un côté, a cherché à bâtir sa légitimité à partir de référents exclusivement économiques et qui, de l'autre, a nié la spécificité du politique en recourant, dans une perspective de gestion, à une série de raccourcis d'ordre juridique - tant autour de la définition même de la « grève ${ }^{7}$ » qu'autour de la légitimation des injonctions et de l'adoption d'une loi spéciale. Elle nous a placé-e-s devant une manifestation particulière de ce que Paul Ricœur a défini, dans Histoire et vérité, comme le "paradoxe politique » propre aux sociétés modernes. Celui-ci tient tout entier, pour Ricœur, dans un décalage insurmontable entre l'idéalité abstraite du concept d'État et la réalité effective du pouvoir, de la force et de la domination. "Nous rêvons », écrit le philosophe, "d'un État où serait résolue la contradiction radicale qui existe entre l'universalité visée par l'État et la particularité et l'arbitraire qui l'affecte en réalité ; le mal, c'est que ce rêve est hors d'atteinte » (Ricoeur, [1955] 1967 : 308).

6. À l'hiver 2012, la hausse prévue des droits de scolarité a d'ailleurs été confirmée et défendue par les membres concernés de la classe politique sur la base d'un prétendu « consensus » dont la consensualité s'est rapidement trouvée démystifiée et dont, par extension, la crédibilité a été complètement minée.

7. La notion de «boycott » sera mise en jeu dès la première semaine de grève, en février 2012 ; les détracteurs du mouvement en généraliseront l'usage au cours du mois de mars. Voir, à ce sujet, la chronologie des événements que Michaël Fortier a contribué à établir, dans Le printemps québécois. Une anthologie, sous la direction de Maude Bonenfant, Anthony Glinoer et Martine-Emmanuelle Lapointe. 
Sur un deuxième plan, l'échec en est également un au sens du principe de représentation politique. Pour justifier certaines de ses décisions ainsi que pour minimiser l'importance de la grève étudiante, le Parti libéral, qui était alors au pouvoir, a eu recours, de façon récurrente, à la notion de «majorité silencieuse », un instrument rhétorique du discours politique que l'on voit apparaître, semble-t-il, dans les années $1960^{\circ}$. Selon Jean Baudrillard, cette notion, dont il rappelle qu'elle est une « simulation » à l'horizon de laquelle « le social a disparu », est ce lieu où, écrit-il, " s'abîme le politique comme volonté et représentation » (Baudrillard, 1982 : 25, 28). Son efficacité ou, mieux, sa « rentabilité » politique vient de ce qu'elle prête un discours au silence tout en faisant de celui-ci le lieu d'inscription même de la volonté collective. Elle opère de cette façon une fictionnalisation de la volonté et, par conséquent, débouche sur une sorte de degré zéro de la représentativité politique. Elle fait échouer le principe de représentation tel que l'entend Cornelius Castoriadis lorsqu'il écrit, en adoptant un point de vue normatif, que la représentation signifie « que nous accordons, dans notre seul intérêt », une délégation de pouvoir à un ou une mandataire qui, dès lors, "n'existe comme tel que pour exprimer la volonté du représenté » (Castoriadis, 1999: 189; c'est l'auteur qui souligne). C'est d'ailleurs autour du concept de représentation, en son double sens politique et sémiologique, que la relation entre champ politique et champ du langage se fait la plus étroite. Tout comme le concept de " masse », dont Baudrillard montre qu'il est un " trou noir où le social s'engouffre », la majorité silencieuse est un signe vide : ce n'est donc pas une catégorie sociologique ni une catégorie démographique, mais plutôt une notion proprement rhétorique (Baudrillard, 1982 : 9). C'est une nébuleuse sans référent dont l'effet est précisément de suspendre la fonction référentielle du langage. La manipulation politique de la notion de majorité silencieuse conduit donc, sur le plan discursif, à cet intéressant paradoxe d'un langage politique qui se cherche des référents sociaux pour asseoir sa représentativité alors même que l'absence de référentialité fait de lui un discours qui ne représente à proprement parler que lui-même. Comme le sondage, entendu comme mécanisme de production de l'« opinion publique », le recours à la notion de majorité silencieuse active une logique qui relève de la simulation et non de la représentation. Comme lui, il repose sur l'implicite discutable et indiscuté selon lequel les opinions, toujours déjà produites et à « portée de tous » et toutes, seraient à la fois existantes, argumentativement fondées et à l'abri des transformations (Bourdieu, [1984] 2002 : 222). Devant la « majorité silencieuse », le discours politique fonctionne comme l'inconscient freudien : il ne connaît pas la négation. II actualise ce préjugé illocutoire selon lequel ne pas dire, c'est consentir.

Sur un troisième plan, cet échec de la représentation politique est donc en même temps un échec au sens de la représentation langagière de la crise étudiante à travers les principaux canaux du discours public. Si, reprenant le modèle normatif de Jürgen Habermas, l'on assigne pour fonction première à l'espace public, comme espace « engendré par l'activité communicationnelle » (Habermas, 1997 : 388), de constituer une caisse de résonance de la vie collective destinée à formuler des problèmes politiques pour en permettre une synthèse élaborée, deux constats s'imposent avec une certaine évidence. D'une part, la concentration du discours autour des enjeux économiques a eu pour effet général d'éclipser le traitement des enjeux annexes (politiques, sociaux et philosophiques) et sous-jacents à la crise étudiante - et cette remarque vaut tout autant, dans l'ensemble, pour le discours des associations et fédérations d'associations étudiantes elles-mêmes. D'autre

8. Ainsi Jean Baudrillard peut-il écrire, en 1982, que « tous les systèmes actuels fonctionnent sur cette entité nébuleuse, sur cette substance flottante dont l'existence n'est plus sociale, mais statistique, et dont le seul mode d'apparition est celui du sondage » (Baudrillard, $1982: 25)$. 
part, une analyse détaillée de certaines strates du discours social permettrait sans doute de faire apparaître « [l]es dominances et [l]es récurrences » qu'un survol même sommaire peut identifier, à savoir l'agglutinement du discours autour de points de condensation thématiques au croisement desquels s'est élaborée une sorte de petit imaginaire social du radicalisme étudiant (Angenot, 2006 : 7). En effet, le discours sur la grève a donné lieu, dans le camp de ses détracteurs, à une double rhétorique : une rhétorique de «la rue », qui a déployé le champ connotatif du vacarme et de la délinquance ${ }^{9}$, lequel mobilisait une variante du vieux thème de l'insécurité que la presse du xix ${ }^{e}$ siècle avait lié au grouillement sourd de la criminalité urbaine (Kalifa, 1995), et une rhétorique de la "violence et de l'intimidation », qui inscrivait elle aussi, en capitalisant sur un réseau de significations momentanément réunis, l'idée même de contestation dans le circuit sémantique du désordre et de l'illégitimité. Ces stratégies rhétoriques ont produit des discours dont l'efficacité vient de ce qu'ils jouent, pour parler en termes sémiologiques, sur les signifiants de façon à les faire passer pour référentiels alors même que les référents, donc la réalité extérieure au langage, se trouvent enterrés par les signifiés. Telles pourraient être, d'ailleurs, les conditions minimales pour que l'on puisse parler de la formation, nécessairement temporaire et située, d'un « imaginaire » opératoire : le fait qu'il soit possible, à un moment donné de la vie collective, d'observer la cristallisation et la circulation d'un réseau cohérent et structuré de signifiés. Pareil réseau produit alors, à plus ou moins grande échelle, une illusion référentielle ${ }^{10}$ dont l'effet est d'opérer - j'en formule l'hypothèse - une sorte d'« irréalisation » de la réalité sociale au sens où Judith Butler décrit le discours pornographique comme «le texte de l'irréalité du genre » (Butler, 2004 : 99).

Mais l'insistance rhétorique sur la « violence » et l'« intimidation », qui a surtout été une appropriation sémantique du « carré rouge » par le discours libéral, attire également l'attention vers un autre aspect politique que la crise étudiante dans son ensemble a permis de révéler. En effet, elle a montré, à la fois et d'un seul tenant, la validité heuristique et les limites de la célèbre définition wébérienne de l'État, l'appareil étatique étant représenté, pour Max Weber, par cette « communauté humaine qui à l'intérieur d'un territoire déterminé revendique pour elle-même et parvient à imposer le monopole de la violence physique légitime ", c'est-à-dire essentiellement policière, militaire et pénale (Weber, 2003 : 118; c'est l'auteur qui souligne). La portée explicative du modèle wébérien, pour être riche, demeure nécessairement limitée : comme l'a déjà montré Bourdieu, l'idée de « légitimité » qui implique celle de reconnaissance, suppose une dimension symbolique (et non seulement physique) qui reste, dans la sociologie classique, insuffisamment théorisée (Bourdieu, 2012 : 204). À ce titre, les événements du printemps 2012 sont particulièrement riches d'enseignement : ils montrent que le monopole étatique de la violence physique légitime ne peut en fait avoir cours que s'il se double d'un travail de légitimation proprement discursif, d'un effort constamment répété pour parvenir à conserver le monopole de la définition légitime, donc publiquement véhiculée et reconnue comme valable, du concept même de «violence ». La légitimation d'un pouvoir sur les

9. On rappellera, par exemple, certaines interventions publiques du ministre de la Justice alors en fonction, JeanMarc Fournier. Celle du 22 mai 2012, rapportée dans un article du Devoir, articule de façon synthétique et exemplaire l'ensemble des éléments discutés dans mon article: «Ce n'est plus du tout une question étudiante, ce n'est plus du tout une question de frais de scolarité, a-t-il affirmé. C'est une question de démocratie. Et on ne peut pas demander à l'État de s'effacer complètement au profit de la rue, au profit du vacarme et du vandalisme. »

10. Selon l'expression du théoricien de la littérature Michael Riffaterre, l'«illusion référentielle », qui naît de ce que le signifié "s'interpose entre les mots et les référents », se rapproche d'une forme d'erreur logique : « Tout comme l'illusion intentionnelle substitue à tort l'auteur au texte, l'illusion référentielle substitue à tort la réalité à sa représentation » (93). 
personnes et les choses passe donc nécessairement par une (tentative de) domination exercée sur les mots.

\section{《 IL FAUT INTERDIRE LA DÉSOBÉISSANCE CIVILE ॥}

Devant ce tissu d'échecs, le devoir de réflexion que la crise étudiante nous laisse en héritage ne peut s'accomplir que si l'on insiste d'abord sur la nécessité qu'il y a de sortir d'un certain discours politique à partir d'un discours sur le politique, qui seul permet de donner un sens aux événements. Il faut opposer à une diabolisation de la contestation, dénoncée comme radicale, une conception radicale de l'action politique. Dans les pages qui précèdent, un certain nombre d'idées ont été proposées et de nombreux problèmes ont été soulevés. Pour terminer, et en guise de récapitulation, je me pencherai sur un cas particulier de façon à intégrer, tout en les articulant, les principaux points d'analyse qui ont été esquissés jusqu'ici.

La notion de "désobéissance civile » a fait, au printemps 2012, une apparition controversée dans le discours public. Tour à tour mobilisée, défendue et stigmatisée, elle n'a pourtant, chez les actrices et acteurs sociaux qui l'employaient pour se positionner dans l'espace politique, fait l'objet d'aucune définition rigoureuse : son introduction dans le discours, au chapitre d'une lutte pour la définition légitime des moyens de lutter démocratiquement, s'est donc effectuée au prix d'une importante décontextualisation sémantique - qui équivaut, pour ainsi dire, à une déconceptualisation. Arrachée à la tradition philosophique où elle s'enracine pourtant, la « désobéissance civile » a joué le rôle d'un révélateur et a mis en relief la distance infranchissable qui sépare deux conceptions radicalement adverses de la démocratie, de même que les positions sociales d'énonciation que ces conceptions manifestent. À cet égard, le discours tenu par l'ex-ministre de la Justice Jean-Marc Fournier, en mai 2012, alors que les tensions atteignent leur paroxysme, paraît symptomatique. Qualifiant publiquement les invitations à la désobéissance d'appels antidémocratiques et antisociaux, il les condamne sévèrement et souligne de surcroît, dans le cadre d'interventions médiatisées, la nécessité qu'il y aurait d'« interdire la désobéissance civile ». Cette dernière déclaration, en forme de boutade involontaire, repose évidemment sur une contradiction patente. Elle trahit, d'un côté, une conception de la démocratie fondée exclusivement sur la croyance en une légitimité sans faille du principe de représentation, donc qui fait correspondre l'expression légitime de la volonté politique à une procédure électorale conventionnalisée. D'un autre côté, elle repose sur le rêve d'une souveraineté étatique qui serait sans reste ni résidu, sur le rêve d'un pouvoir qui serait sans extériorité et qui aurait pleine maîtrise sur des sujets politiques qu'il réduirait à l'état de sujets de la politique, au sens où Althusser disait que le pouvoir interpelle l'individu en sujet, donc au sens d'un assujettissement.

Or, dès que l'on confronte cette version déconceptualisée de la « désobéissance civile » comme action « antidémocratique » et " antisociale » (au sens où elle peut être perçue comme rupture d'un contrat) à celle qui fait l'objet d'une conceptualisation philosophique, il devient possible, voire nécessaire, de considérer que, dans certaines situations critiques, la mise en péril du ressort démocratique trouve bien davantage sa source dans l'obéissance inconditionnelle que dans les formes concertées et réfléchies de la désobéissance civile : "Indépendamment de ce qui est à chaque fois l'objet du litige, implicitement la désobéissance civile réclame toujours que la forma- 
tion constitutionnelle de la volonté politique soit en phase avec les processus de communication de l'espace public » (Habermas, 1997 : 411). Cette définition, proposée par Habermas dans Droits et démocratie, indique que le recours à la désobéissance constitue en lui-même la reconnaissance et la critique d'un décalage existant entre le légal et le légitime. Elle fournit du même coup à la désobéissance civile son fondement rationnel et sa vocation publique et nous conduit au cœur d'une tension conceptuelle qui mérite d'être maintenue et, en un sens, cultivée : la perspective habermassienne nous invite à renverser la conception mise de l'avant par le ministre Fournier et à parler, paradoxalement, d'un « droit » à la désobéissance civile. Mais ce « droit » fonctionne, ici, sans référence légale. II s'agit d'un droit au sens politique de l'expression, au sens très précisément où la désobéissance civile fait appel, comme le suggère Habermas, à une justification d'ordre morale, métajuridique ou constitutionnelle.

Hannah Arendt a largement insisté, pour sa part, sur la dimension irréductiblement collective de la " désobéissance civile », élargissant ainsi une notion qui, chez Henry David Thoreau, demeurait prise dans une acception individualiste : «Celui qui fait acte, énonce-t-elle, de désobéissance civile ... agit au nom et en faveur d'un groupe particulier. II lance un défi aux lois et à l'autorité établie à partir d'un désaccord fondamental et non parce qu'il entend personnellement bénéficier d'un passe-droit » (Arendt, [1969] 1972: 77). En fait, le «droit » en quelque sorte paradoxal que désigne, en creux, la théorie habermassienne ne peut exister que s'il prend appui sur la dimension collective de la contestation, qui lui fournit ainsi son cadre de référence. II ne peut, comme l'indique la position arendtienne, être synonyme d'impunité dans la mesure, justement, où il inspire une action qui ne repose, pour l'individu, sur aucune prétention à être exempté des sanctions légales prévues pour telle ou telle infraction commise. Car c'est précisément la connaissance et la reconnaissance de l'existence du système légal qui donnent à la désobéissance civile ses motivations et son mode d'existence propre, elle qui n'existe, comme moyen d'une lutte politique pour la reconnaissance, que parce qu'il y a toujours déjà interdiction légale de désobéir. La désobéissance civile s'alimente à même un vide. Elle vit précisément de ce qui vise à lui retirer le droit de vivre. À travers le traitement qu'elle propose de la notion de « désobéissance civile», la philosophie politique énonce finalement la nécessité d'opérer une séparation de principe entre les deux sphères non superposables du politique et du juridique, une séparation qui, pendant la grève étudiante, a été largement niée par le discours politique - c'est même cette dénégation qui, en quelque sorte, se trouve à l'origine de la « crise » dont j'ai parlé.

Or, à l'évidence, cette séparation ne peut être obtenue et protégée que sur la base d'une acceptabilité politique de cette séparation elle-même, toute lutte pour la définition légitime du concept de démocratie étant en même temps, plus profondément, une lutte pour la définition et la délimitation du champ politique lui-même. À l'intérieur de ce champ, il devrait être possible d'éviter le court-circuitage juridique par la mise en place de dispositifs agonistiques de discussion, qui demeurent les seuls à offrir, dans le cadre d'une société plurielle établie sur un fonctionnement parlementaire, la possibilité d'une articulation manifeste entre volonté et représentation. C'est ce que tend à faire apparaître, contextuellement, une analyse même sommaire du traitement réservé à la notion de "désobéissance civile », déchirée entre la signification que lui a fait prendre sa circulation dans le discours médiatique et dans la discussion théorique dont elle fait l'objet dans le cadre du discours philosophique. 
Cette idée d'une autonomie du champ politique a été développée de façon prometteuse, me semble-t-il, par Judith Butler. En soulignant le rôle constitutif que joue l'usage et l'appropriation des mots dans les luttes sociales et politiques, c'est-à-dire dans le jeu des identifications individuelle et collective, Butler montre le risque que l'emprise juridique fait potentiellement courir à la revendication politique. Or, la portée et l'ampleur de la grève étudiante lui viennent précisément, pour une certaine part au moins, de n'avoir bénéficié d'aucune reconnaissance juridique. Sans cette absence de reconnaissance, qui exigeait du mouvement qu'il invente, nomme et construise sa propre légitimité, son expansion et son extension, donc sa (re)traduction sous la forme d'une lutte pour la définition légitime du concept de démocratie, n'auraient pu être possibles. "Penser » la crise, comme j'en faisais au départ la proposition, c'est donc refuser de restreindre le débat autour de la question du fonctionnement des universités ; c'est aussi clamer et réclamer l'autonomie de la sphère politique, reconnaître et accepter le fait fondamental que des concepts litigieux puissent demeurer vulnérables aux réappropriations et aux résignations. Et si, en effet, toute crise politique est appelée à se constituer comme telle dans le champ des significations, à l'intérieur duquel toute irrévocabilité sémantique a disparu, alors le travail sur le concept même de démocratie constitue, dans le contexte politique actuel et à l'issue du printemps 2012, l'un des principaux ressorts démocratiques à exploiter. En effet, cette « absence de toute irrévocabilité est à l'origine d'un dilemme interprétatif précieux, car elle suspend le besoin d'un jugement définitif pour affirmer au contraire que le langage est vulnérable à la réappropriation. Cette vulnérabilité marque la façon dont l'exigence démocratique postsouveraine se manifeste sur la scène d'énonciation contemporaine » (Butler, 2004 : 131) 
Angenot, Marc. Théorie du discours social. Notions de topographie des discours et de coupures cognitives. Montréal : Presses de l'Université McGill, 2006. Imprimé.

Arendt, Hannah. Du mensonge à la violence. Essais de politique contemporaine. 1969. Paris : Calmann-Lévy, 1972. Imprimé.

---. Qu’est-ce que la politique? 1993. Paris : Seuil, 1995. Imprimé.

Baudrillard, Jean. À l'ombre des majorités silencieuses ou la fin du social. Paris : Denoël/Gonthier, 1982. Imprimé.

Bonenfant, Maude, Anthony Glinoer et Martine-Emmanuelle Lapointe, dir. Le Printemps québécois. Une anthologie. Montréal : Écosociété, 2013. Imprimé.

Bourdieu, Pierre. Questions de sociologie. 1984. Paris : Minuit, 2002. Imprimé.

---. Sur la télévision suivi de L’Emprise du journalisme. Paris : Raisons d’agir, 2008. Imprimé.

---. Sur l'État. Cours au Collège de France 1989-1992. Paris : Seuil, 2012. Imprimé.

Butler, Judith. Le Pouvoir des mots. Discours de haine et politique du performatif. Trad. Charlotte Nordmann. Paris : Éditions Amsterdam, 2004. Imprimé.

Castoriadis, Cornelius. Figures du pensable. Paris : Seuil, 1999. Imprimé.

Dumont, Fernand. Le Lieu de l'homme. 1968. Montréal : Bibliothèque Québécoise, 2005. Imprimé.

Dupuis-Déri, Francis. Démocratie. Histoire politique d'un mot aux États-Unis et en France. Montréal : Lux Éditeur, 2013. Imprimé.

Freitag, Michel. Le Naufrage de l'université et autres essais d'épistémologie politique. 1995. Québec: Nota Bene, 1998. Imprimé.

Gauchet, Marcel. La Religion dans la démocratie. Parcours de la laïcité. Paris: Gallimard, 1998. Imprimé.

Habermas, Jürgen. Droit et démocratie. Entre faits et normes. Trad. Rainer Rochlitz et Christian Bouchindhomme. Paris: Gallimard, 1997. Imprimé.

Hartog, François. Régimes d'historicité. Présentisme et expériences du temps. 2003. Paris : Seuil, 2012. Imprimé.

Kalifa, Dominique. L'Encre et le sang. Récits de crime et société à la Belle-époque. Paris : Fayard, 1995. Imprimé.

Mouffe, Chantal. Le Politique et ses enjeux. Pour une démocratie plurielle. Paris : La Découverte, 1994. Imprimé.

Ricoeur, Paul. Histoire et vérité. 1955. Paris : Seuil, 1967. Imprimé.

---. Temps et récit. 1. L'intrigue et le récit historique. Paris : Seuil, 1983. Imprimé. 
Riffaterre, Michael. «L’illusion référentielle. » 1978. Littérature et réalité. Paris : Seuil, 1982. 91-118. Imprimé.

Sauvageau, Florian, et Simon Thibault. «Sommet sur l'enseignement supérieur - Les médias ont-ils failli à la tâche?» Le Devoir 20 février 2013 : http://www.ledevoir.com/politique/quebec/371318/les-mediasont-ils-failli-a-la-tache. Web.

Veyne, Paul. Comment on écrit l'histoire. Essai d'épistémologie. Paris : Seuil, 1971. Imprimé.

Weber, Max. Le Savant et le politique. Trad. Catherine Colliot-Thélène. Paris : La Découverte, 2003. Imprimé. 